\title{
Partial 2-Stage Revision in Chronic Hip Arthroplasty Infections: A Review
}

\author{
Francesco Castagnini'*, Luca Busanelli', Giovanni Bracci', Enrico Tassinari', Federico Biondi', Claudio \\ Masetti ${ }^{1}$, Aldo Toni ${ }^{1}$
}

${ }^{1}$ Hip and Knee Surgery, Istituto Ortopedico Rizzoli, Bologna, Italy

*Corresponding Author: Francesco Castagnini, M.D., Hip and Knee Surgery, Istituto Ortopedico Rizzoli, Via Pupilli

1, 40136 Bologna, Italy. Tel: +39-0516366148, Email: francescocastagnini@ hotmail.it

Received January 13, 2018; Accepted May 3, 2018; Online Published May 20, 2018

\begin{abstract}
Background: Periprosthetic hip infections (PHIs) are troublesome complications of hip arthroplasties. The gold standard procedure for treating chronic PHI is a 2-stage approach. Recently, however, more conservative approaches have been developed to spare the osseointegrated components and avoid sequestra, bone loss, devascularization, and difficult reconstructions. The partial two-stage approach, which leaves the well-fixed component in situ and removes the loosened component, may be an effective strategy.

Objective: This paper, a narrative mini-review, analyzed the preliminary results of a partial 2-stage approach to treating chronic hip arthroplasty infections.

Methods: Pertinent papers describing the partial 2-stage approach (leaving the well-fixed component in situ and removing the loosened component) were collected and evaluated.

Results: Six main case series were selected. A total of 76 patients were included. Many patients were treated with socket removal and stem retention with quite similar surgical techniques. Many cases included highly virulent bacteria, and no pre-operative selection about comorbidities was performed. The first outcomes of this approach were promising, with a rate of infection control ranging from $81.3 \%$ to $100 \%$ at mid-term follow-up.

Conclusion: This approach proved good at mid-term follow-up; however, many concerns still exist. In particular, the indications are imprecise, and the role of biofilm is still unclear. Despite the first good outcomes, the partial 2-stage approach for chronic PHI should be validated by multicenter prospective studies.

Keywords: Hip Arthroplasty, Infection, Two-Stage
\end{abstract}

\section{Background}

Periprosthetic hip infections (PHIs) are still troublesome complications after primary implants, occurring in $0.3 \%$ $2.9 \%$ of cases. ${ }^{1}$ The gold standard treatment for chronic $\mathrm{PHI}$ is the 2-stage approach, consisting of implant removal and spacer positioning, subsequent antimicrobial therapy, and reimplantation in a second surgical step., ${ }^{1,2}$ Using this approach, the overall rates of infection control were reported to be positive $(63 \%-100 \%))^{2}$ However, many drawbacks had to be faced, such as prolonged antibiotic therapy and the high costs related to the 2 stages; a few cases were not revised due to death or poor medical condition. ${ }^{2}$ Thus, lower rates of success and patient satisfaction were reported. ${ }^{2}$

A single-stage approach was developed to overcome some of the previously-mentioned drawbacks. ${ }^{3}$ Some reports on the single-stage procedure in late chronic PHI described positive results, in line with the 2-stage approach. ${ }^{3}$ Nevertheless, the best outcomes were achieved in patients with few comorbidities. ${ }^{3}$ The narrow indications were good local tissues with viable vascularization, low virulence bacteria, and "healthy" patients. ${ }^{3}$ Moreover, these 2 procedures (one-stage and 2 -stage) required implant removal, resulting in damage to bone and soft tissue. ${ }^{3}$ To avoid devascularization, sequestra, and consequent difficult reconstruction, highly retentive procedures with one- or 2-stage approaches were developed.

The highly retentive one-stage approach was described by El-Husseiny and Haddad. ${ }^{4}$ In their study, 18 patients with no debilitating diseases and no sinus tracks were treated with a super-selective one-stage procedure, removing the modular parts and the loosened component. The reimplantation took place in the same surgical step, and it was followed by a high-dosage antibiotic therapy in the immediate post-operative period. The authors treated resistant and highly virulent bacteria as well. Three patients out of the initial 18 failed due to septic relapses. The clinical outcomes were satisfying. The authors emphasized that

Copyright (C) 2018 The Author(s). This is an open-access article distributed under the terms of the Creative Commons Attribution License (http:// creativecommons.org/licenses/by/4.0), which permits unrestricted use, distribution, and reproduction in any medium, provided the original work is properly cited. 
such a technique should be limited to very selective cases. A similar experience was reported by Ji et al, who achieved good infection control (87.1\%).

Despite the positive outcomes, the previous technique had some limitations related to non-healthy patients, non-susceptible microorganisms, and previous surgical scars and sinus tracks, which were considered as possible contraindications or factors leading to septic relapse., Thus, a partial 2-stage approach was developed to treat more complex cases, retaining the well-fixed components. ${ }^{6-12}$

\section{Objective}

The aims of this mini-review were to evaluate the partial 2-stage approach in the treatment of chronic PHI and assess the surgical technique, the clinical results, and the rate of infection control.

\section{Methods}

A narrative mini-review was designed. The electronic database PubMed was searched for articles on the partial 2-stage approach in chronic PHI. Only papers published in the English language and before January 2018 were selected. The following predetermined keywords were used: "partial" or "retain" or "exchange" or "selective" together with "infection" or "hip" or "revision". The persistence of chronic PHI was determined after the selected papers were carefully read. The abstracts of the selected papers were screened and hard copies of pertinent articles were obtained. The reference lists of the included papers were also examined to search for related works. Case reports were excluded.

\section{Results}

Fourteen papers were initially selected, but only 6 were considered eligible for the study as they dealt strictly with the 2-stage approach in chronic PHI (Table 1). ${ }^{6-11}$ The surgical procedure described in each paper was evaluated. The most frequently adopted techniques and practical tricks are summarized in the subsequent section. The results and the limits of the 6 studies are discussed and summarized in the section entitled "Clinical Results".

\subsection{Surgical Technique}

There was a wide consensus regarding the surgical procedure. $^{6-11}$ In all main case series, the preoperative evaluation of patients consisted of serological analyses, a hip joint aspiration, and radiological assessment. The loose component was carefully assessed using serial X-rays and CT scans. The use of nuclear scanning was inconstant.

In the first surgical stage, an accurate debridement, a synovectomy, and a modular part removal were performed. ${ }^{6-11}$ Multiple tissue samples were retrieved to isolate the bacteria. The loose component was removed. The supposed well-fixed component was visually inspected and manually probed; in some cases, an invasive attempt to remove the stable component was performed with a dedicated instrumentation (Explant, Zimmer, Warsaw; USA). ${ }^{7}$ If the component proved to be well-fixed, it was retained. Then the exposed metal parts were scrubbed and an aggressive lavage was performed. Antibiotic powders or disinfectants were often added. ${ }^{9,10}$ In case of stem retention, an antibiotic-loaded cement head was then prepared; vancomycin, tobramycin, or gentamycin were the most preferred antimicrobial agents to be mixed with high viscosity cement. The cement head was hand-molded on a metal head. Sometimes a dedicated instrumentation was used (StageOne Hip Cement Spacer Molds and StageOne Select Hip Cement Spacer Molds, Biomet, Warsaw, Indiana). ${ }^{10}$ The implant was then reduced and a suction drainage was positioned. In case of stem removal, a handmade femoral spacer was positioned inside the femoral canal. $^{7}$

A frequent complication after the first stage was spacer dislocation. The authors recommended a proper restoration of offset and leg length in order to provide a stable implant..$^{6-11}$

After the first stage, an antimicrobial therapy was prescribed, based on the microbial findings and the patient's comorbidities and allergies. ${ }^{6-11}$ The therapy was orally or intravenously administered under the control of an infectious disease consultant. Usually, a 4-6-week therapy was recommended, until the serological markers (C-reactive protein and sedimentation rate) became negative. Afterwards, a hip joint aspiration could be performed to determine whether bacteria were still present; however, this practice seemed to be not strictly necessary. ${ }^{8}$

Then, the second surgical stage took place. ${ }^{6-11}$ The spacer was removed and multiple samples were taken. An intraoperative histopathological examination was performed to exclude a septic relapse/persistence. The

Table 1. The Selected Papers Describing a 2-Stage Approach in chronic PHI

\begin{tabular}{|c|c|c|c|c|c|c|c|}
\hline Authors & Year & $\begin{array}{l}\text { No. of } \\
\text { Cases }\end{array}$ & $\begin{array}{l}\text { Highly Virulent or } \\
\text { Resistant Bacteria }\end{array}$ & Spacer Use & $\begin{array}{l}\text { Mean Follow- } \\
\text { up in Months }\end{array}$ & $\begin{array}{c}\text { Percentage of } \\
\text { Infection Control }\end{array}$ & Notes \\
\hline Anagnostakos et al & 2010 & 12 & Yes & Yes & 55 & 92 & Cemented components \\
\hline Lee et al & 2013 & 17 & Yes & Yes & 48 & 88 & Few acute cases \\
\hline Ekpo et al & 2014 & 19 & Yes & Yes & 40 & 83 & $\begin{array}{l}\text { Failures had multiple } \\
\text { surgeries }\end{array}$ \\
\hline Lombardi et al & 2014 & 7 & Yes & Yes & 19 & 86 & Very high body mass index \\
\hline Fukui et al & 2016 & 5 & Yes & Yes & 50 & 100 & Two hemi-arthroplasties \\
\hline Chen et al & 2017 & 16 & Yes & $\begin{array}{l}\text { Yes and no } \\
\text { (cup) }\end{array}$ & 60 & 81 & One cup retention \\
\hline
\end{tabular}


reconstruction was performed and the new component was positioned; bone grafts were used if necessary.

\subsection{Clinical Results}

To date, according to the literature, 76 patients have been treated with the partial 2-stage approach (6 papers). The percentage of infection eradication ranged from $81.3 \%$ to $100 \%$ at mid-term follow-up. ${ }^{12}$ Six main case series should be discussed in detail (Table 1).

Anagnostakos et al reported on 12 cases; septic cup loosening and stable stem fixation were evident in each patient. $^{6}$ After cup removal and antibiotic-loaded cement head positioning, the patients were treated with antimicrobial therapy. Then reimplantation was performed after a mean of 88 days. At 55 months after reimplantation, 91.6\% of patients were free from infection. One patient died due to cardiopulmonary infection. The present case series also included highly virulent bacteria.

Lee at al described 19 patients with chronic or acute hematogenous PHI and well-fixed stems. ${ }^{8}$ In all cases, the authors removed the acetabular socket and implanted an antibiotic-loaded cement head. Highly virulent bacteria were isolated. A 4-6-week antibiotic therapy was performed, until the serologic parameters normalized. In the second stage, reimplantation took place; morselized bone grafts were used when necessary. Two patients with no signs of infection did not undergo the second stage. Seventeen patients were reimplanted, and 15 of them had not experienced a septic relapse after 4 years. The 2 patients with septic relapses were immunocompromised and critically ill (diabetes, cirrhosis, and carcinoma).

A similar experience was described by Ekpo et al in a mixed population of 19 primary and revision arthroplasties with chronic PHI and well-fixed stems, the authors performed an analogous surgical technique. ${ }^{9}$ Highly virulent and resistant bacteria were also isolated. In their study, $11 \%$ of cases experienced a septic relapse at 4 years, and 3 patients died after the reimplantation. The failed cases had multiple previous surgeries and isolations of resistant bacteria.

Lombardi et $\mathrm{al}^{10}$ reported about 7 patients treated with a similar technique described by Ekpo et al. ${ }^{9}$ Six of the 7 patients were considered free from infection. The mean Harris Hip Score (HHS) was 72 at a mean follow-up of 1.6 years.

Fukui et al followed 5 patients with PHI limited to the socket. ${ }^{11}$ The cups were removed and the cementless stems were left in situ. An antibiotic-loaded spacer was positioned in every case. Highly resistant bacteria were isolated. After high-dosage antibiotic therapy, the reimplantation took place. After 4.2 years, all patients were free from infection. The mean HHS score was 86 points.

Chen et al reported a case series of 16 patients with $\mathrm{PHI}$ and a single loose component. ${ }^{7}$ A partial 2-stage approach was selected. In case of cup removal, the spacer was an antibiotic-loaded cement head; in the case of stem removal and cup retention ( 1 case), an antibiotic-loaded cement spacer was positioned in the femoral canal. Resistant bacteria were isolated. The rate of infection control was $81.3 \%$ at a mid-term follow-up. Three patients failed, and all of them were immunocompromised, critically ill, or prone to infections (systemic lupus erythematosus, end stage renal disease, diabetes mellitus, drug abuse).

\section{Discussion}

The partial 2-stage approach yielded positive outcomes at mid-term follow-ups. ${ }^{6-12}$ Based on the retention of wellfixed components, this approach reduced sequestra, bone loss, devascularization, and simplified the reimplantation stage. However, this novel approach has been highly criticized. ${ }^{12,13}$

One main issue was probably related to patient selection and the presence of biofilm. ${ }^{12,13}$ As all reported case series using the 2-stage approach were retrospective, the roles of biofilm and patient selection were questionable. The removal of all components was believed to be paramount in chronic PHI in order to avoid septic recurrence: in fact, most of the infections were present in the biofilm state, which was built up over a period of 36 hours to 3 weeks. ${ }^{13}$ Thus, the partial approach contradicted previous knowledge about chronic PHI. However, the retrospective design of the partial approach case series may have been confusing, selecting infections without biofilm (if they existed). Moreover, a few authors emphasized that septic relapses after partial the 2-stage approach were more frequent in cases of immunocompromised or ill patients, patients with resistant bacteria, and patients with multiple previous surgical procedures. ${ }^{6-11}$ Other authors excluded patients with sinus tracks, as this clinical condition was related to severe infections. ${ }^{6-11}$ Due to the small number of patients, it was difficult to provide satisfying selective criteria.

The issues related to the surgical technique were mainly 2: the use of spacers and bone grafts. ${ }^{6-14}$ Spacers were used when the neck had to be protected (thus, stem retention in non-dual taper implants) and when lack of soft tissue quality and bone stock deficiency were present. In the case of the 2-stage approach, the use of bone grafts was not discouraged, as the previous debridement stage and the antimicrobial therapy provided sufficient sterilization. Lee et al reported no additional risks of septic relapse in cases of bone grafting. ${ }^{8}$

Another concern was related to the antimicrobial therapy. ${ }^{12,13}$ Unfortunately, a uniform protocol was not developed. The antimicrobial therapy was administered based on antibiotic resistance, medical condition, availability, and allergies. A 4-6-week therapy was usually adopted, generally under the control of an infectious disease consultant. The aim of the antimicrobial therapy was to normalize the serological markers. ${ }^{6-13}$

\section{Conclusion}

The partial 2-stage approach has been proven effective in preliminary papers; however, more studies about the 
partial 2-stage approach are needed before any conclusions are drawn. Recently, Liebs proposed the use of a registry to record the number and the follow-up of partial 2-stage cases. ${ }^{13}$ Unfortunately, registries may provide unreliable portrayals of outcomes. Patients unwilling to perform a second stage with a sterilized hip may be considered failures; conversely, infected patients unwilling to remove the implant after a failed partial 2-stage approach would be considered successes. Thus, multicenter, prospective studies are the first step towards validating this procedure.

\section{Authors' Contributions}

All authors contributed equally to the work, collecting and analyzing data $(\mathrm{GB}, \mathrm{ET}, \mathrm{FB}, \mathrm{CM})$, and preparing the manuscript (FC, LB, AT).

\section{Conflict of Interest Disclosures}

The authors declare that they have no conflicts of interest.

\section{Ethical Approval}

Ethical approval for this study was not necessary due to the nature of the manuscript.

\section{References}

1. Kuzyk PR, Dhotar HS, Sternheim A, Gross AE, Safir O, Backstein D. Two-stage revision arthroplasty for management of chronic periprosthetic hip and knee infection: techniques, controversies, and outcomes. J Am Acad Orthop Surg. 2014;22(3):153-164. doi:10.5435/jaaos-22-03-153.

2. Berend KR, Lombardi AV Jr, Morris MJ, Bergeson AG, Adams JB, Sneller MA. Two-stage treatment of hip periprosthetic joint infection is associated with a high rate of infection control but high mortality. Clin Orthop Relat Res. 2013;471(2):510-518. doi:10.1007/s11999-012-2595-x.

3. Wolf $\mathrm{M}, \mathrm{Clar} \mathrm{H}$, Friesenbichler J, et al. Prosthetic joint infection following total hip replacement: results of one-stage versus two-stage exchange. Int Orthop. 2014;38(7):1363-1368. doi:10.1007/s00264-014-2309-y.

4. El-Husseiny M, Haddad FS. The Role of Highly Selective
Implant Retention in the Infected Hip Arthroplasty. Clin Orthop Relat Res. 2016;474(10):2157-2163. doi:10.1007/s11999-0164936-7.

5. Ji B, Xu B, Guo W, et al. Retention of the well-fixed implant in the single-stage exchange for chronic infected total hip arthroplasty: an average of five years of follow-up. Int Orthop. 2017;41(5):901-909. doi:10.1007/s00264-016-3291-3.

6. Anagnostakos K, Jung J, Kelm J, Schmitt E. Two-stage treatment protocol for isolated septic acetabular cup loosening. Hip Int. 2010;20(3):320-326. doi:10.1177/112070001002000305.

7. Chen KH, Tsai SW, Wu PK, Chen CF, Wang HY, Chen WM. Partial component-retained two-stage reconstruction for chronic infection after uncemented total hip arthroplasty: results of sixteen cases after five years of follow-up. Int Orthop. 2017;41(12):2479-2486. doi:10.1007/s00264-017-3505-3.

8. Lee YK, Lee KH, Nho JH, Ha YC, Koo KH. Retaining well-fixed cementless stem in the treatment of infected hip arthroplasty. Acta Orthop. 2013;84(3):260-264. doi:10.3109/17453674.20 13.795830 .

9. Ekpo TE, Berend KR, Morris MJ, Adams JB, Lombardi AV, Jr. Partial two-stage exchange for infected total hip arthroplasty: a preliminary report. Clin Orthop Relat Res. 2014;472(2):437448. doi:10.1007/s11999-013-3168-3.

10. Lombardi AV Jr, Berend KR, Adams JB. Partial two-stage exchange of the infected total hip replacement using disposable spacer moulds. Bone Joint J. 2014;96-b(11 Suppl A):66-69. doi:10.1302/0301-620x.96b11.34360.

11. Fukui K, Kaneuji A, Ueda S, Matsumoto T. Should well-fixed uncemented femoral components be revised in infected hip arthroplasty? Report of five trial cases. J Orthop. 2016;13(4):437442. doi:10.1016/j.jor.2015.09.006.

12. Yan Z, Fang R, Liu T. Partial component-retained two-stage reconstruction for chronic infection after uncemented total hip arthroplasty: good or bad? Int Orthop. 2018;42(3):729-730. doi:10.1007/s00264-017-3748-z.

13. Liebs TR. CORR Insights((R)): The Role of Highly Selective Implant Retention in the Infected Hip Arthroplasty. Clin Orthop Relat Res. 2016;474(10):2164-2167. doi:10.1007/s11999-0164987-9.

14. Marczak D, Synder M, Sibinski M, Wasko M, Polguj M, Kowalczewski J. Comparison of patients treated with or without a spacer in two stage revision hip arthroplasty for periprosthetic joint infection. J Infect. 2016;73(5):520-522. doi:10.1016/j. jinf.2016.09.006. 